\title{
Subacute Low Back Pain: infectious spondylodiscitis with epidural and psoas abscess
}

\author{
Judit Bartkó*1, Daniella Ladóczky-Hulló ${ }^{2}, V$ iktor Petrovszki ${ }^{3}$, Katalin Varga ${ }^{1}$ \\ ${ }^{1}$ Csongrád-Csanád County Health Care Centre-Hódmezővásárhely-Makó, Hódmezővásárhely, Hungary \\ ${ }^{2}$ Faculty of Medicine, Department of Rheumatology and Immunology, University of Szeged, Szeged, Hungary \\ ${ }^{3}$ Department of Radiology, Albert Szent-Györgyi Health Centre, Szeged, Hungary
}

Received: December 7, 2020

DOI: $10.5430 /$ crim.v7n $4 \mathrm{p} 10$
Accepted: December 16, 2020 Online Published: January 5, 2021

URL: https://doi.org/10.5430/crim.v7n4p10

\begin{abstract}
We describe a case of infectious spondylodiscitis of the lumbar spine complicated by epidural and bilateral psoas abscesses which started with subtle symptoms and a non-specific clinical picture. Diagnosis is based on clinical, laboratory and radiological features. It can be difficult and often delayed due to the rarity of the disease and the high frequency of low back pain in the general population. Our aim is to raise awareness for the possibility of specific low back pain which requires quick and indispensable action from the physician.
\end{abstract}

Key Words: Spondylodiscitis, Low back pain, Infection of spine, Psoas abscess, Epidural abscess, Specific back pain

\section{INTRODUCTION}

Specific low back pain accounts for only a minority of low back pain cases (approx. 15\%). ${ }^{[1]}$ Infectious spondylodiscitis is one of the rare, possible causes. Diagnosis is based on clinical, laboratory and radiological features. ${ }^{[2]}$ However, recognition can be difficult due to a non-specific initial clinical presentation. Our aim is to raise awareness for this serious condition which requires quick and targeted antibiotic treatment.

\section{Case presentation}

A 51 year-old man presented with a one-month history of mild-moderate low back pain to our rheumatology clinic. His pain radiated vaguely and predominantly into his left leg and was accompanied by a tingling sensation on the dorsal aspect of his left foot. A few weeks ago, he received local paraverebral infiltrations with betamethasone injection twice at a private practice and took diclofenac, omeprazole, tizanidine and vitamine B1 orally. However, his low back pain was not relieved by rest and medications. He did not experience any leg weakness, had no loss of bowel or bladder control. His temperature was normal and had suffered no trauma. He denied any chest, abdominal and urinary tract symptoms. His general condition was good. He is a physical worker and he experienced episodes of back pain in the recent years, for which he used aceclofenac occasionally. His past medical history included tonsillectomy, treated hypertension and hyperlipidemia.

Physical examination of the patient revealed obesity and that the lower lumbar vertebral column was moderately painful to pressure. Lumbar spinal movements were restricted only by $1 / 3$ and were mildly-moderately painful. Straight leg raise test was negative on both sides. There was no neurological

\footnotetext{
*Correspondence: Judit Bartkó; Email: drbartkojudit@gmail.com; Address: Csongrád-Csanád County Health Care Centre-Hódmezővásárhely-Makó, Hódmezôvásárhely, Hungary.
} 
symptom other than paresthesia on the dorsum of his left foot, I. and II. toes. Hip movements were normal. He also had a swelling on the mid-distal third of the dorsal aspect of his right forearm with diffuse redness. The swelling was not painful to touch. His General Practitioner decided to put him on amoxicillin/clavulanic acid to treat this lesion the day before. Upon questioning, he mentioned that he might have bad teeth. Based on the clinical picture, there was a suspicion of infection, and therefore, a diagnostic search was initiated.

Right elbow and wrist X-ray showed no bone structure alterations, surgical consultation excluded cellulitis and thrombophlebitis. Lumbar CT scan was ordered because his low back pain was resistant to therapy. His initial laboratory tests showed the following notable results: WBC: $13.58 \mathrm{G} / \mathrm{l}$, Hct: 0.38 L/L, Plt: 394 G/1, creatinine: 111 micromol/1, ALP: 349 U/l, gGT: 81 U/l, GPT: 81 U/1, Fe: 7.1 micromol/1, Transferrin: 1.59 g/l, Ca: 2.78 mmol/1, Prot: 72 g/l, CRP: $41 \mathrm{mg} / 1$, Antistreptolysin titre: $357 \mathrm{U} / \mathrm{l}, \mathrm{RF}: 23 \mathrm{IU} / \mathrm{ml}$, ANA: neg, ANCA: neg., CA 19-9: normal, CEA: normal, PSA: normal, , Protein electrophoresis: no monoclonal component, urine protein: $1+$, urine leukocyte: negative. Otorhinolaryngology, urology, chest X-ray, abdominal and pelvic ultrasound examinations, stool blood tests $(3 \mathrm{x})$ were all negative.

Changing his NSAID medication and maximizing the daily dose did not ease his low back pain. By the end of a oneweek course of amoxicillin/clavulanic acid therapy, the lesion on his right forearm subsided. However, he developed non-itching purpuras on his extremities and in both gluteal regions. Dermatology consultation suggested a drug hypersensitivity reaction and by omitting the suspected medications, his exanthems started to heal as well.

In the meantime, the CT result (see Fiure 1) showed irregular bone structure in the endplate of the LIV. vertebra and also irregular bone structure and partially missing bone structure in the upper two-third of LV. vertebra suggesting either spondylodiscitis or lytic bone disease.

Urgent neurosurgery referral was made. The neurosurgeon suspected infectious spondylodiscitis. He started the patient on oral clindamycin $600 \mathrm{mg}$ QDS and recommended contrast-enhanced magnetic resonance imaging to be done on the lumbar spine as soon as possible.

Meanwhile, the patient's low back pain got worse, described as throbbing by the patient and his general condition started to decrease as well. He still had normal body temperature. Urgent laboratory workup (two weeks after the initial tests) revealed normal WBC (8.48 G/1), CRP did not improve (46 $\mathrm{mg} / \mathrm{l})$, creatinine worsened (129 micromol/l).
The patient was admitted to the inpatient unit of the Rheumatology Department for intravenous clindamycin therapy. Contrast-enhanced MRI of the lumbar spine (see Figures 2 and 3) showed extensive infection of the lower lumbar spine with bilateral psoas abscess $(1.5 \mathrm{~cm}$ and $4 \mathrm{~cm}$ largest diameter respectively).

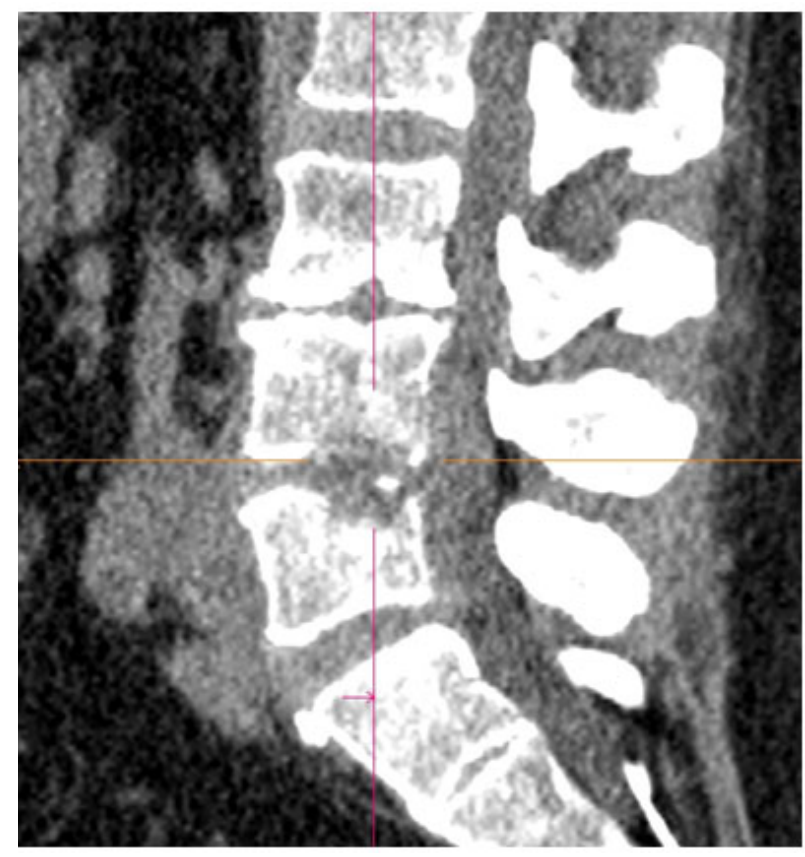

Figure 1. CT sagittal view: LIV. infectious spondylodiscitis

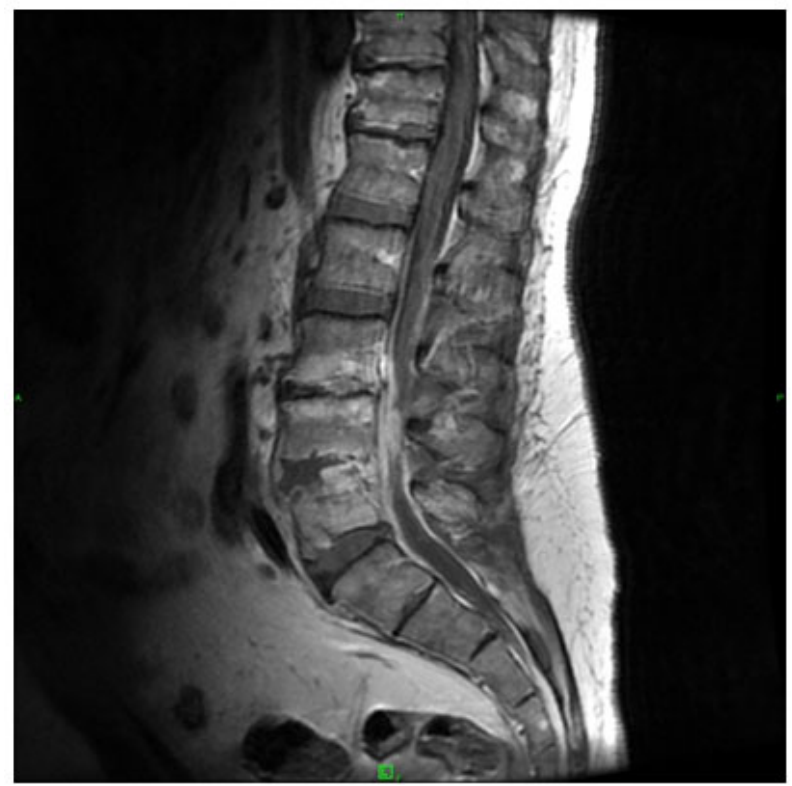

Figure 2. MRI, contrast enhanced T1 weighted image, sagittal plane: extensive infection originating from LIV-V. segment and involving the epidural space 


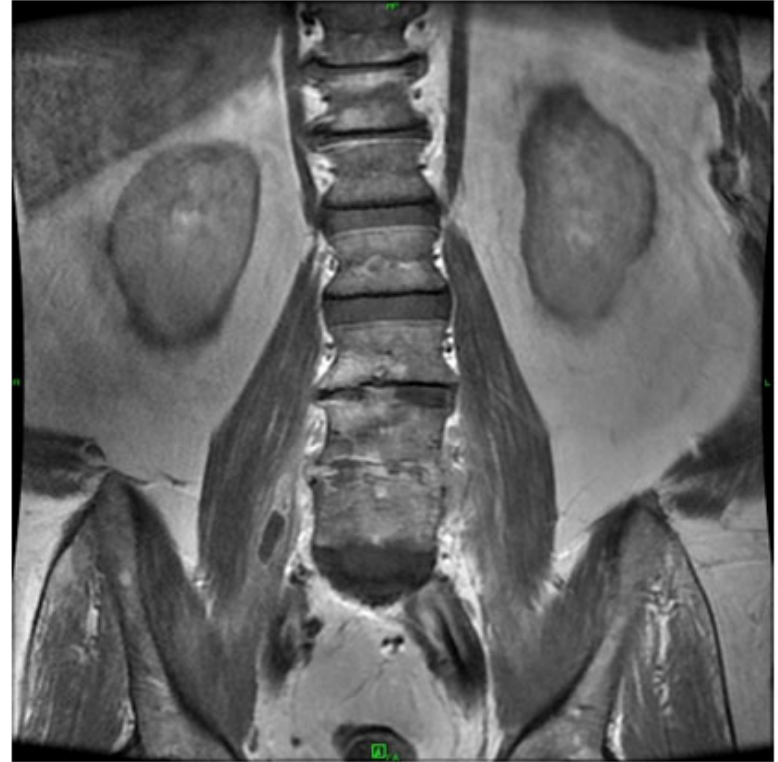

Figure 3. MRI, contrast enhanced T1 weighted image, coronal plane: smaller psoas abscess on the right side

Search for the origin of the infection was continued, urine and throat culture proved to be negative. Oral surgical referral and intervention was made due to spontaneous tooth extraction, but neither wound discharge, nor other focus of infection was found. Blood culture revealed Staphylococcus aureus positivity and ceftriaxone was added to clindamycin in his intravenous treatment regimen. The patient's CRP gradually normalised, he remained apyrexial and the paresthesia on the dorsum of his foot slowly diminished. He mobilised with Boston corset and he was discharged in good general condition after a four-week course of intravenous antibiotic therapy. He continued with oral clindamycin for another four weeks at home and he is currently being followed up at the outpatient clinic.

\section{Discussion}

Low back pain is very common in the general population and its cause is non-specific in most cases ( approx. 85\%), ${ }^{[1]}$ has a musculoskeletal origin and requires only symptomatic treatment. Regarding our patient, the mild-moderate subacute low back pain, which radiated vaguely and mostly into the left leg with radiculopathy, started only with subtle clinical symptoms. However, a specific cause, a serious, lifethreathening condition stood in the background: infectious spondylodiscitis with extensive epidural abscess and bilateral small psoas abscess.

The clinical symptoms of infectious spondylodiscitis are nonspecific. ${ }^{[2-4]}$ Fever is often missing, ${ }^{[2]}$ laboratory findings can be mild. ${ }^{[2-4]}$ Recognition of the condition is difficult and often delayed as a result of its rarity. ${ }^{[2-4]}$ In our case, with special regard to the bilateral psoas abscess, the bilateral paravertebral injections received by the patient a few weeks earlier, are strongly suspected as the root cause. ${ }^{[5]}$

According to literature, Staphylococcus aureus is the most frequent pathogen. ${ }^{[2,4]}$ However, blood cultures are indispensible in order to identify the offending agent. They should be taken before starting antimicrobial therapy, to be able to give targed treatment. In our case, hemocultures were obtained only after initiating oral antibiotics, but they still produced a positive result and the antibiotic treatment was modified.

Due to the adequate intravenous therapy which was started in time, our patient is on the road to recovery now. CRP is a reliable marker for monitoring response to treatment ${ }^{[2,4]}$ and contrast-enhanced MRI is the modality of choice ${ }^{[2]}$ to get a clear radiological picture. The follow-up MRI of our patient shows significant regression regarding the inflammatory signs, further treatment and clinical follow-up is needed.

\section{Conclusion}

A specific cause should be suspected in the case of subacute low back pain which does not respond adequately or worsens to symptomatic treatment or is accompanied by an atypical clinical picture. Therefore, prompt laboratory and imaging studies are necessary. Clinical suspicion is the mainstay of early diagnosis.

\section{CONFlicts OF INTEREST Disclosure}

The authors have declared no conflicts of interest.

\section{REFERENCES}

[1] Almeida DC, Kraychete DC. Low back pain-a diagnostic approach Rev Dor. 2017; 18: 173-7. https ://doi .org/10.5935/1806-0 013. 20170034

[2] Gouliouris Th, Aliyu SH, Brown NM. Spondylodiscitis: update on diagnosis and management. J Antimicrobial Chemother Suppl (3) 2010; 65:11-24. PMid:20876624 https://doi.org/10.1093/ja c/dkq303

[3] Orso V, Serdeira A, Ziegler M, et al. Diagnostic difficulties in bacte12 https ://doi.org/10.1590/S1808-185120151404132593

[4] Naveen P, Souvik P, Gagandeep Y, et al. Evaluation of challenges in diagnosis of spontaneous subacute pyogenic spondylodiscitis in immunocompetent patients: Experiences from a Tertiary Care Center. Asian Spine J. 2019; 13: 621-629. PMid:30966724. https: //doi.org/10.31616/asj.2018.0220

[5] Mavrogenis AF, Megaloikonomos PD, Igoumenou VG, et al. Spondylodiscitis revisited. EFORT Open Rev. 2017; 11: 447-461. PMid:29218230. https://doi.org/10.1302/2058-5241.2.16 0062

ISSN 2332-7243 E-ISSN 2332-7251 